The principal magnetic directions of the molecule are evidently (1) along the line joining the centres of the three benzene rings, (2) along the perpendicular direction in the plane of the rings, and (3) along the normal to the plane of the rings. The susceptibilities along these directions can be calculated from the known values for the benzene molecule (or from those for benzene and for diphenyl molecules) and are found to be

$$
\begin{aligned}
K_{1}=K_{2} & =-91 \\
K_{3} & =-254
\end{aligned}
$$

respectively, in $10^{-6}$ c.g.s. e.m.u. per gm. mol.

The principal susceptibilities of the crystal are, in the same units :

$$
\begin{aligned}
& \chi_{1}=-94 \\
& \chi_{2}=-203 \\
& \chi_{3}=-139 .
\end{aligned}
$$

$\chi_{3}$ is the susceptibility along the $b$ axis; the $\chi_{1}$ axis, in the (010) plane, is found to lie in the acute angle $\beta$, making an angle of $14 \cdot 3^{\circ}$ with the $c$ axis.

From these data the orientations of the two molecules in the unit cell can be deduced in the same manner as was described for diphenyl in a previous communication ${ }^{3}$. It is thus found that the lengths of the molecules lie in the $(010)$ plane, in the acute angle $\beta$, making an angle of $14 \cdot 3^{\circ}$ with the $c$ axis, while the planes of the two molecules are inclined at $+56 \cdot 6^{\circ}$ and $-56 \cdot 6^{\circ}$ respectively to the $(010)$ plane. These values are in good agreement with the values $15 \cdot 3^{\circ}$ and $\pm 56^{\circ}$ obtained for these angles by Miss Pickett from her X-ray data.

K. S. Krishnan.

S. BANERJEE.

210, Bowbazar Street, Calcutta.

Nov. 15.

1 Natore, 130, 313, Aug. 27, 1932 ; and 131, 653, May 6, 1933. Phil. Trans., A, 231, 235; 1933.

${ }^{2}$ Proc. Roy. Soc., A, 142, 333; 1933.

s Phil. Trans., A, 231, 256; 1933.

\section{Reversed Fine Structure of the $\alpha$-Rays}

The fine structure of the $\alpha$-rays, discovered by Rosenblum and studied by him and by Lord Rutherford and his co-workers, was explained by Gamow's supposition that some of the $\alpha$-particles escape from the nuclei with energies smaller than normal by certain discrete quantities. The difference of the energies remains in corresponding nuclei in the form of energy of excitation of other $\alpha$-particles. As such excited $\alpha$-particles are unstable, they fall to the ground-level and radiate the surplus of energy as $\gamma$-quanta.

Such excited $\alpha$-particles can not only fall to the ground-level, but have also a certain probability of penetrating the potential barrier. By this means they give rise to groups of $\alpha$-rays with energies greater than normal by the same quantities by which the energies of the fine structure groups of the preceding nuclei were less than normal. Such reversed fine structure groups can form only a small part of the main group, for all the elements that produce such phenomena (that is, all the elements following those that show fine structure) have comparatively small decay constants, and the probability of escape from the excited levels of nuclei must be much smaller for the excited $\alpha$-particles than the probability of falling to the ground-level with emission of $\gamma$-quanta.

The relative intensity of the 'reversed' group is

$$
N=P \frac{l_{\alpha^{\prime}}}{g(\nu)}
$$

where $P$ is the proportion of the excited nuclei (or the relative intensity of the corresponding fine structure group of the preceding element), $l_{x^{\prime}}$ is the decay constant of the excited $\alpha$-particles (calculated from the Gamow-Houtermans equation) and $g(v)$ is the probability of emission of $\gamma$-quanta of frequency $\nu$, which according to Taylor and Mott is nearly proportional to $V^{3}$ for dipole radiation and to $V^{5}$ for quadrupole radiation.

For example, the fine structure group of An must give the reversed group of AcA with relative intensity $N \sim 0.23 \frac{3.2 \times 10^{3}}{10^{11}}=\sim 0.8 \times 10^{-8}$. (This value was

obtained by assuming for calculating $l_{x^{\prime}}$ that the radius of the excited level is the same as that of the normal one ; as actually it is greater, this value gives only the lower limit.) This intensity is not far beyond the sensitivity of modern methods.

Radium Institut,

A. Poxessitsky.

Leningrad.

Nov. 4.

\section{Change of Orientation of a Barnacle after Meta- morphosis}

IT is known that immediately after settlement, the cypris larvæ of the acom barnacle can move about for a short time before they metamorphose ; and that they tend to collect in cracks along which their long axis is usually orientated. I am not aware, however, of any record of a change of orientation occurring once the larva has metamorphosed.

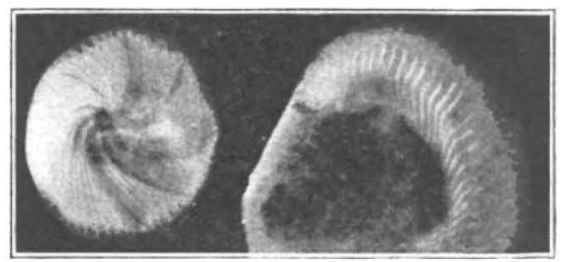

(a)

(b)

Fig. 1. $(a) \times 5 . \quad(b) \times 4$.

I obtained recently a specimen of Balanus improvisus, Darwin, var. assimilis, which was growing in the glass outlet pipe of one of the aquarium tanks here. The base of the barnacle, together with its radially running pores, was clearly visible through the glass (Fig. 1a), and these pores were curved instead of, as normally, straight. Now since each of these pores corresponds with a parietal pore in the walls, and since the basis, which is added to at the edge only, eannot rotate on the substratum to which it is attached, it follows that the lower edges of the walls must have rotated as the animal grew. Further, since the parietal pores in the walls were vertical and not oblique, it follows that the whole wall, up to the apex, must have rotated correspondinglyin this case through about $90^{\circ}$.

Of seven specimens of this variety from the plaice- 